Wu et al., Afr J Tradit Complement Altern Med., (2018) 15 (1): 151-157

https://doi.org/10.21010/ajtcam.v15i1.15

\title{
IMPROVING EFFECT OF PLATYCODIN D ON ETHANOL-INDUCED FATTY LIVER VIA KEAP1-NRF2-ARE SIGNAL PATH
}

\author{
Jingtao $\mathrm{Wu}^{1, *}$, Lei Zhou ${ }^{2}$, Guiwen Yang ${ }^{3}$, Fan Tao ${ }^{4}$, Maoshan Wang ${ }^{4}$, Jianguo $\mathrm{Hu}^{4}$
}

${ }^{1}$ Department of Science and Technology and ${ }^{2}$ School of Biological Science and Technology, University of Jinan, Jinan 250022, China. ${ }^{3}$ School of Life Science, Shandong Normal University, Jinan 250014, China. ${ }^{4}$ Department of Food Science and Nutrition, University of Jinan, Jinan 250022, China.

\begin{tabular}{|c|}
\multicolumn{1}{|c|}{ Article History } \\
\hline Received: April, 17, 2017 \\
Revised Received: Dec. 04, 2017 \\
Accepted: Dec. 05, 2017 \\
Published Online: Dec. 29, 2017
\end{tabular}

\begin{abstract}
Background: Platycodin D (PD) is one of the important active ingredients in the root of the Platycodon grandiflorum (Jacq.) A. DC (PG), a traditional medicine with many pharmacological activities, especially hepatoprotective activity. There are some researches on the mechanism of liver protection, but it is not very clear. It is necessary to investigate it deeply.

Materials and Methods: In order to evaluate the effect of PD on ethanol-induced fatty liver (EFL) and investigate the possible mechanism, the EFL models of rats and BRL-3A cells were set up and treated with PD of $20,30 \mathrm{mg} / \mathrm{kg}$ body weight/day for four weeks, respectively. The hepatic histochemistry, liver function enzymes, oxidative stress levels, antioxidant enzymes and signal path enzymes were assayed.

Results: PD significantly $(P<0.01)$ activates signal path enzymes of Keap1, Nrf2 and small Maf either in vitro or in vivo, increases hepatic antioxidant enzyme expression of HO-1, SOD and GSH-Px, improves the oxidative stress indicated by CYP2E1, ROS, T-AOC capacity and MDA contents, reduces levels of serum ALT and AST, and then improves the liver injury degree by histological evaluation.

Conclusion: PD is an effective inhibitor of EFL to ameliorate liver impairment ethanol-induced via Keap1-Nrf2-ARE signal path.
\end{abstract}

Keywords: platycodin D; ethanol-induced fatty liver; nuclear factor erythroid 2 related factor 2; Kelch-like ECH associated protein 1; small Maf

List of Abbreviations: PD: platycodin D, PG: Platycodon grandiflorum (Jacq.) A. DC, EFL: ethanol-induced fatty liver, Nrf2: Nuclear factor erythroid2 related factor 2, Keap1: Kelch-like ECH associated protein 1, ARE: antioxidant response element, HO-1: heme oxygenase1, SOD: superoxide dismutase, GSH-Px: glutathione peroxidase, CYP2E1: Cytochrome P450 2E1, T-AOC: total antioxidant capacity, MDA: methane dicarboxylic aldehyde, ROS: reactive oxygen species.

\section{Introduction}

Improper ethanol consumption can cause ethanol-induced fatty liver (EFL), the most common one of the liver impairments (Arteel, 2003). The major cause of it is the role of lipid peroxidation and oxidative stress (Vishnudutt et al., 2008) caused by the CYP2E1-generated ROS (Butura, et al., 2009). And it has been shown that the diseases-related oxidative stress has a decline of Nrf2 expression (Sussan et al., 2009). Nrf2 is a member of the Cap'n'Collar transcription factor family, and normally bound to the Kelch-like ECH associated protein (Keap1) in cytoplasm (Tong et al., 2006). Disassociated from Keap1, the Nrf2 enters into nucleus and dimerizes with small Maf proteins, then recognizes and binds to antioxidant response element (ARE) in promoter of target genes to promote the transcriptional activation of antioxidative genes and cytoprotective enzymes, which forms the defense system against oxidative stress (Yates et al., 2006).

Ideal pharmacological reagents that can prevent or reverse the EFL are the traditional natural medicines (Ngo et al., 2013). So PD, extracted from Platycodon grandiflorum (Jacq.) A. DC (as shown in Figure 1) with many medical effects in traditional Chinese medicine system (National pharmacopoeia commission, 2015), was chosen to evaluate the 
effect on EFL in rats and BRL-3A cell. The liver protection of PG and its extracts was reported in the literature, such as saponins' hepatoprotective effects on acute ethanol-induced in mice (Khanal et al.,2009), aqueous extract' improving effects on acetaminophen or carbon tetrachloride-induced liver damage in mice(Lee et al., 2001, Lee and Jeong, 2002), protective effects of saponins against fatty liver in chronic ethanol feeding via the activation of AMP-dependent protein kinase(Khanal et al.,2009), treatment with PD effectively against EFL through anti-inflammatory and anti-endotoxic process via TLR4-MyD88-NF- $\alpha \mathrm{B}$ signal path (Wu et al., 2016). On the other hand, the saponins, oleanolic acid compounds from natural products can protect liver by increased expression of liver Nuclear factor erythroid 2 related factor 2 (Nrf2) and its downstream protective enzymes (Reisman et al., 2009; Sussan et al., 2009). Therefore, in this article, we further studied whether the effect of PD on EFL is controlled by the other mechanism of Nrf2-related signal path.

\section{Materials and Methods Preparation of PD}

The PD used in this study is the oleanolic acid, a triterpenoid bidesmoside compose of an aglycone moiety, 3-glucose and 28-O-arabinosyl-rhamnosyl-xylosyl-apiosyl (as shown in Figure 2), purity of 92.28\% and relative molecular mass of 1225.38, a polar solvent extract obtained from the roots of PG that possessed two years' growth time, collected from Lu-mountain (Zibo, China), and identified by the expert of plant taxonomy, Professor Shoujin FAN from College of Life Sciences, Shandong Normal University (Jinan, China). The methods of extracting and identifying of PD has been published previously (Wu et al., 2012).

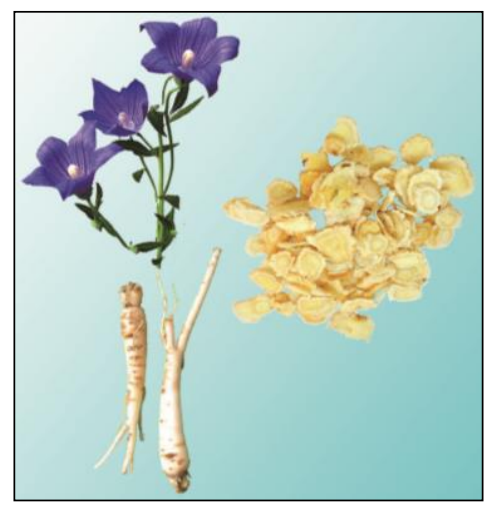

Figure 1: Images of PG

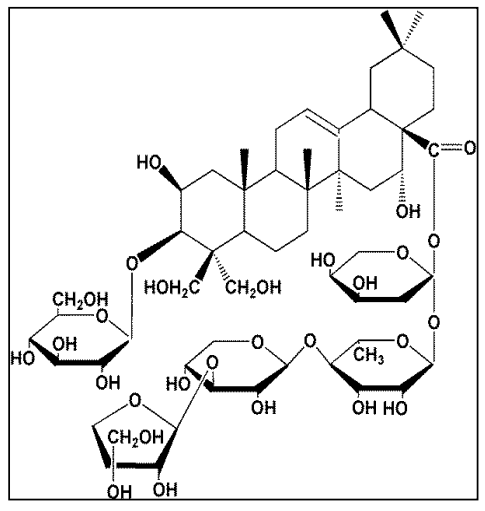

Figure 2: Structure of PD

\section{Animals and treatments}

The sixty experimental Female Sprague-Dawley rats weighing $(210 \pm 28) \mathrm{g}$ were randomly divided into five groups $(n=12)$ : normal control (NC) group, ethanol-induced fatty liver (EFL) group, low dose PD (PD-1) group, high dose PD (PD-h) group and positive medicine simvastatin (PM) group, respectively. Except NC group was given equal normal saline during the entire experiment, the other rats were given $40 \%$ ethanol $10 \mathrm{~mL}$ and fish oil (Mission Hills, CA 91346-9606, America) $4 \mathrm{~mL} / \mathrm{kg}$ BW/day by gavage with needle tubing, respectively, for five weeks, so as to induce the EFL. From the sixth to the ninth week, PD-l and PD-h groups were given 20 and $30 \mathrm{mg} / \mathrm{kg} \mathrm{BW/d} \mathrm{PD} \mathrm{by}$ gavage, and PM group was given $25 \mathrm{mg} / \mathrm{kg}$ BW/d simvastatin (Beijing shuanglu, China, H20058534), respectively. All experiments were indeed carried out in accordance with the guidelines of the Ethics Committee on Animal Experiments of Medical School of Shandong University (NO. 2010011).

The blood sample from heart of each rat anaesthetized with intraperitoneal sodium pentobarbital was centrifuged 
at $3000 \mathrm{r} / \mathrm{min}$ to obtain serum. The liver samples from rats sacrificed by cervical dislocation were rapidly removed, washed in saline, and immersed in liquid nitrogen.

\section{Cell culture and treatments}

The BRL-3A cells (Chinese Academy of Sciences, China) were seeded onto 96-well plates and divided into 5 groups $(n=4)$ : group CG treated with 5\% Tween 80 in PBS which was the solvent for PD, group EG treated with 200 $\mathrm{mmol} / \mathrm{L}$ ethanol, group PDl treated $0.15 \mathrm{mg} / \mathrm{mL} \mathrm{PD}$, group PDh treated with $0.25 \mathrm{mg} / \mathrm{mL}$ PD, and group SIM treated with $0.25 \mathrm{mg} / \mathrm{mL}$ simvastatin. The latter three groups co-treated with $200 \mathrm{mmol} / \mathrm{L}$ ethanol. The treatment duration was $24 \mathrm{~h}$. PD and simvastatin were added $2 \mathrm{~h}$ before the ethanol treatment.

\section{Biochemistry assay of hepatic function and oxidation levels}

The biochemical indicators of rats were measured using ALT and AST commercial test kits (Sigma, USA). Hepatic oxidation levels of rats were determined by T-AOC, MDA content and ROS commercial kits (Nanjing Jiancheng, China).

\section{Histological observation assay of liver}

The freezing liver tissues were processed routinely to $10 \mu \mathrm{m}$ sections (Leica-cm3050s, Germany). The sections were immersed in $10 \%$ formalin for $30 \mathrm{~min}$, washed with $70 \%$ ethanol, and immerged in Sudan III dye for 25 min. The $70 \%$ ethanol was added on them to wash again. At last the sections were counterstained with hematoxylin for 5 min. The microphotographs were taken by microscope (CX51, Olympus, Japan) to investigate the extent of ethanol-induced steatosis and deposition.

\section{ELISA assay of Keap1 protein expression}

The liver tissue was homogenised in RIPA buffer (Shanghai Beyotime, China), centrifuged at $12000 \mathrm{r} / \mathrm{min}$ for 10 min at $4{ }^{\circ} \mathrm{C}$, and the cytokines levels in the supernatant were measured by ELISA kits (BioLegend, America) and BCA protein assay Kits (Thermo Fisher, America).

\section{Immunohistochemical analysis of Nrf2 protein expression}

Antibody against Nrf2 (Wuhan Boster, China) was dropped onto the $8 \mu \mathrm{m}$ section of liver, incubated at the $37^{\circ} \mathrm{C}$ for $1 \mathrm{~h}$. The secondary antibody was added to the sections at $37^{\circ} \mathrm{C}$ for $20 \mathrm{~min}$, and then the reagents of strept-avidin-biotin complex were added to the sections at $25{ }^{\circ} \mathrm{C}$ for $20 \mathrm{~min}$. The target protein was stained and the resultant photomicrographs were taken with microscope camera (Cx51, Olympus Japan) and quantitative by software of Evaluation Image-Pro ${ }^{+} 4.5$.

\section{Immunoblot analysis of CYP2E1 protein expression}

The $40 \mu \mathrm{g}$ lysed proteins of liver tissue were loaded onto a 12\% sodium dodecyl sulphate-polyacrylamide gel and transblotted onto a polyvinylidene fluoride membrane (Bio-Rad, America). Nonspecific proteins were blocked for $2 \mathrm{~h}$. The membranes were immunoblotted with primary antibody of CYP2E1 (1:800, Cell Signaling Technology, America) at $4{ }^{\circ} \mathrm{C}$ overnight, then incubated with a secondary goat anti-rabbit horseradish peroxidase-conjugated antibody (Zhongshan Golden bridge, China) for $2 \mathrm{~h}$, and finally detected by chemiluminescent substrate kits (Alpha Diagnostic, America), following by autoradiographic and densitometric analysis.

\section{Real-time PCR analysis of Nrf2-related genes expression}

Total RNAs samples obtained from each liver and BRL-3A cells were isolated by TRIZOL reagents (Invitrogen, America) and the cDNA synthesis were done using cDNA synthesis kits (Bio-Rad, America). LightCyler Probe Design software 2.0 was used to design primers for the target genes of the Nrf2, Keap1, small Maf, HO-1, SOD and GSH-Px, and reference gene GAPDH. Applied Biosystems 7900HT Fast Real-Time PCR System (ABI, America), TOYOBO-Realtime PCR Mater Mix (SYBR Green, Toyobo, Japan), standard procedures, and SDS software 2.3 were used to assess the mRNAs expression. The $\mathrm{Ct}$ values were calculated by the computer automatically, and the data were calculated and counted with $2^{-\Delta \Delta^{\mathrm{Ct}}}$ methods.

\section{Statistical analysis}

All date were presented as (mean $\pm \mathrm{SD}$ ). Differences among groups were assessed using unpaired student's $t$ test and one-way ANOVA. The significantly different at ${ }^{* *} P<0.01$ and ${ }^{*} P<0.05$ compared with AFL or EG groups, ${ }^{\#} P<$ 0.01 compared with $\mathrm{NC}$ or $\mathrm{CG}$ groups. 


\section{Results}

\section{Changes of ethanol-induced liver injury and oxidative stress}

The histopathological studies showed that EFL group, compared to the NC's, were induced degeneration in hepatocytes and hepatic cords, and focal necrosis. The severe hepatic lesions induced by ethanol plus fish oil were reduced remarkably $(P<0.01)$ by the administration of PD and simvastatin, and this was in good agreement with the results of ALT and AST (as shown in Figure 3). The levels of serum ALT and AST in EFL rats were significantly increased by $232.39 \%, 323.59 \%$, respectively, compare to the NC group $(P<0.01$, as shown in Figure 4$)$. The oxidative stress reflected by T-AOC, MDA, CYP2E1 and ROS levels were significantly $(P<0.01)$ higher by $73.17 \%$, and lower by $180.66 \%, 319.80 \%$ and $182.37 \%$, respectively, in EFL rats than those in normal control group. Similarly, The HO-1, SOD and GSH-Px levels are lower by $68.82 \%, 61.35 \%, 81.65 \%$, respectively, in EFL rats than them in NC rats $(P$ $<0.01$, as shown in Figure 5).

The marked improving effects $(P<0.01)$ on liver injury, biochemical criterion, oxidative stress and oxidase activity appeared in PD and simvastatin treatment rats compared with EFL group's.
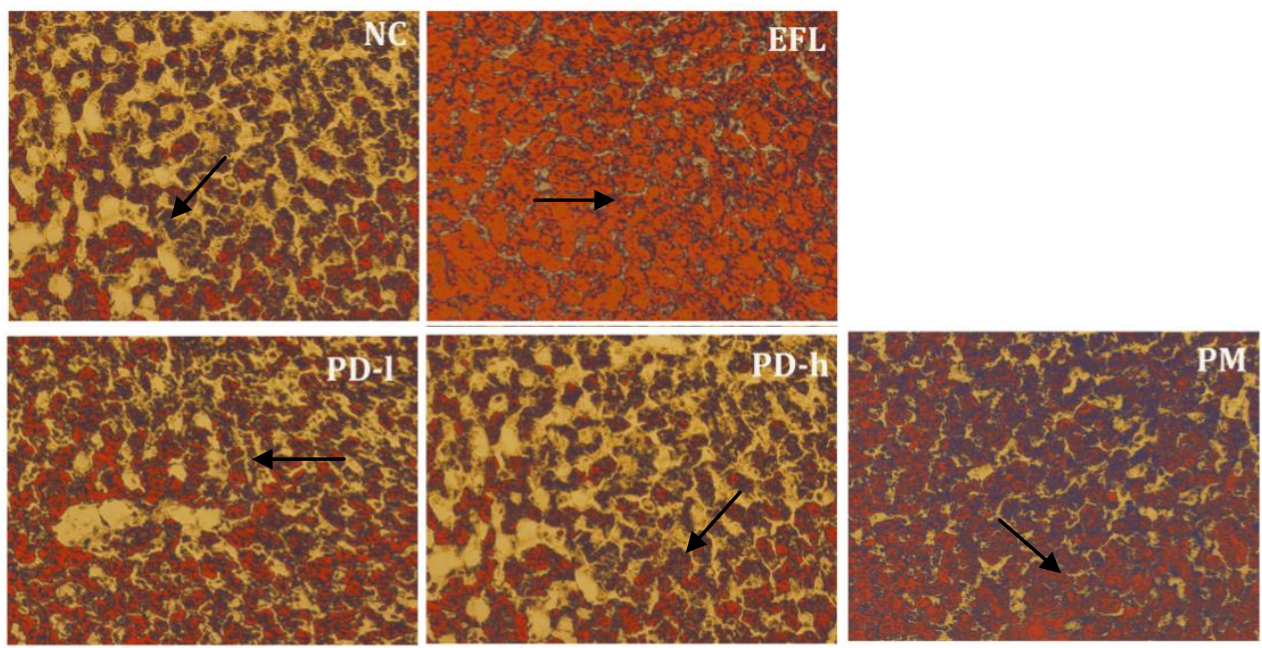

Figure 3: Images of histological injury on liver of rats

5 images were randomly selected in each group and were randomly selected 3 horizons at high magnification $(x 400)$ to view.
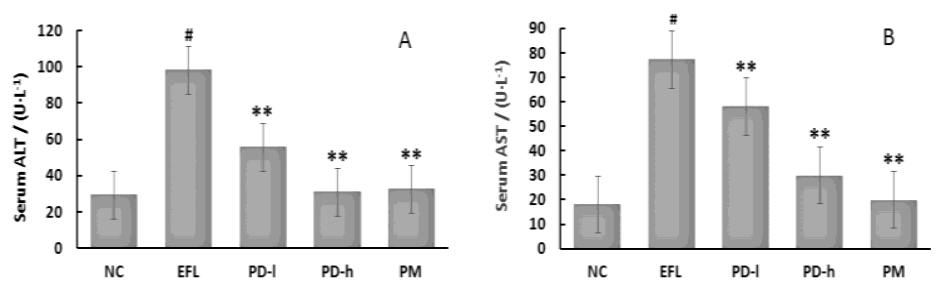

Figure 4: Changes of liver injury in rats.
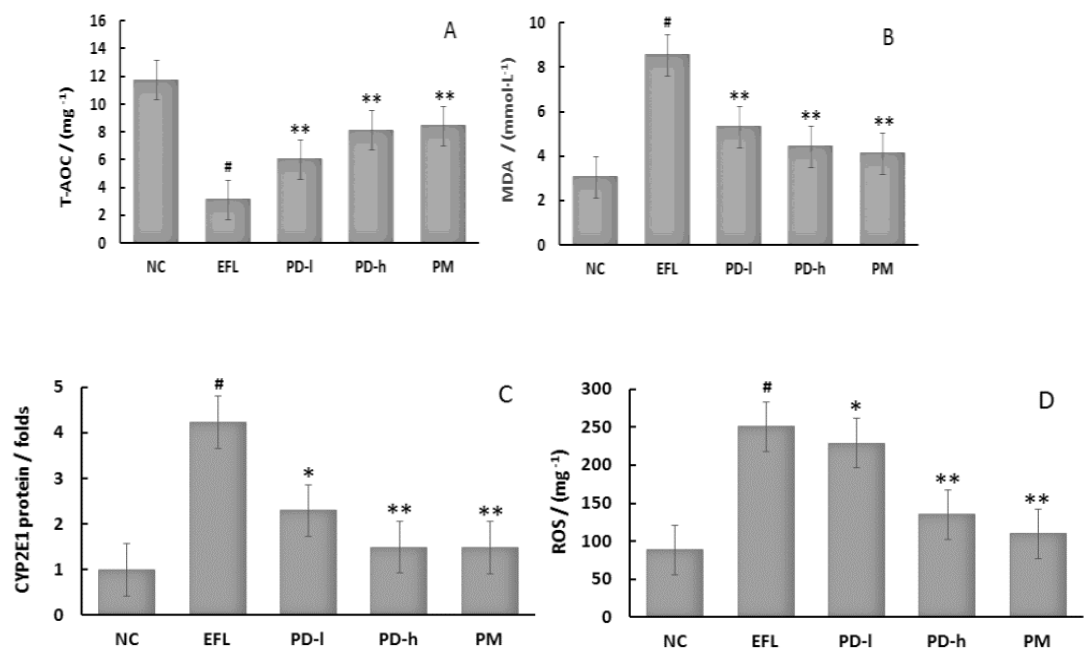

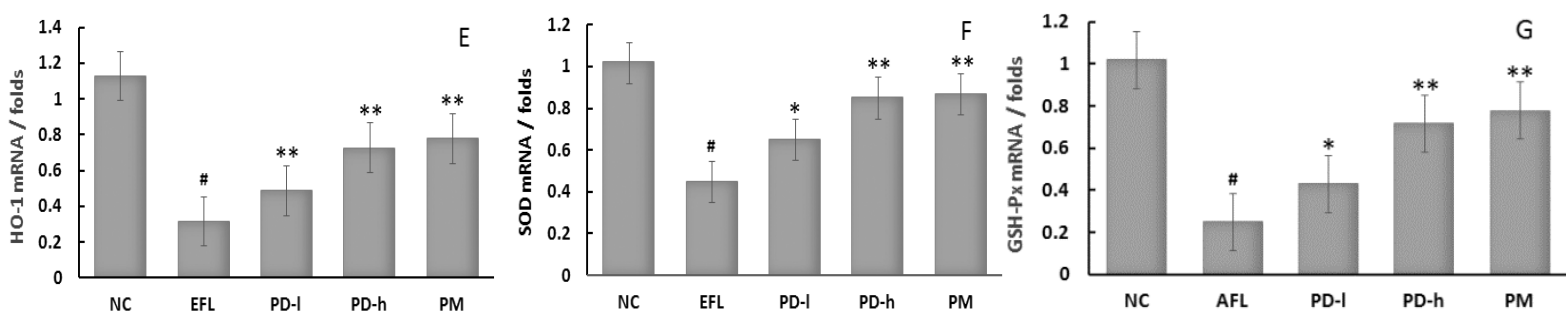

Figure 5: Changes of oxidative stress on liver of rats

\section{Changes of Nrf2-related signal path enzymes}

The proteins expression of Keap1 and Nrf2, and the mRNA expression of small Maf were decline dramatically $(P<0.01)$ by $69.78 \%, 63.04 \%$ and $55.88 \%$ respectively in EFL rats compared to normal control group's (as shown in figure 6). Ethanol treatment to BRL-3A cells decreased the three mRNA expression obviously $(P<0.01)$ by $60.12 \%$, $69.89 \%$ and $58.36 \%$, respectively, in EG model group cells compared with CG group's (as shown in figure 7). Either in vitro or in vivo, the reductions were significantly $(P<0.01)$ increased by PD and simvastatin treatments.
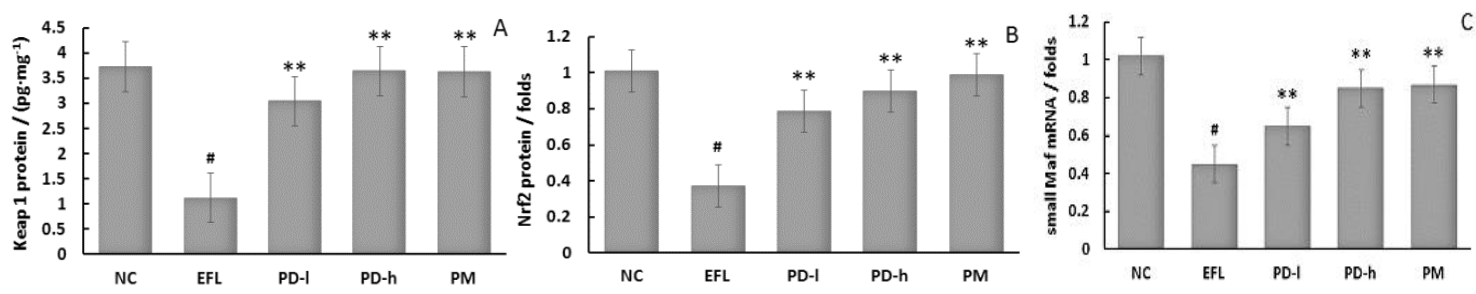

Figure 6. Changes of Nrf2-related signal path enzymes of rats
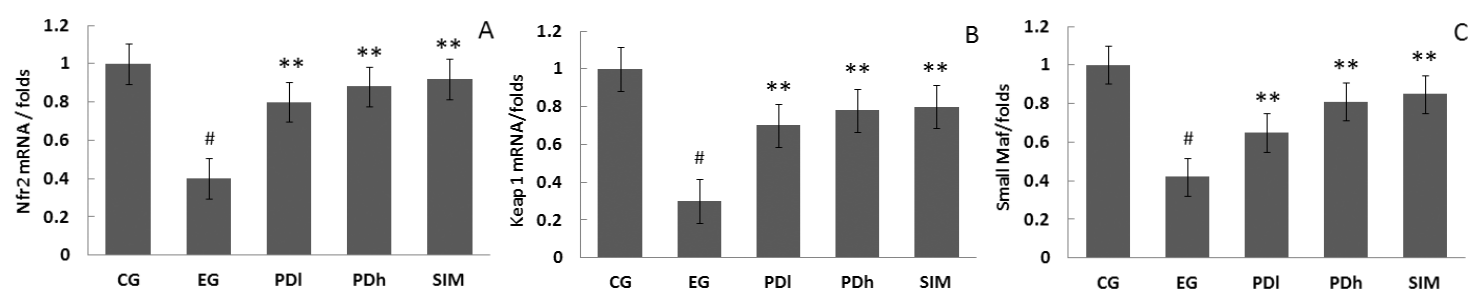

Figure 7: Changes of Nrf2-related signal path enzymes of BRL-3A cells

\section{Discussion}

Experimental model of EFL was set up successfully by gavage of ethanol plus fish oil for five weeks. In our published articles (Wu et al, 2016), except the histological observation, ALT levels and AST levels assay, the other biochemical parameters such as serum total bilirubin and endotoxin, and liver TG assays confirmed that. Our results showed that obvious hepatic injury was observed in experimental rats and the model could mimic key aspects of alcoholic liver disease of human's.

PD is one of the saponins, and has a property to lyse blood cells, so we have adopted oral feeding way for rats instead of intravenous one. The doses of experimental verification of 20 and $30 \mathrm{mg} / \mathrm{kg} \mathrm{BW} / \mathrm{d}$ PD to rats were not seen the cases of drug toxicity during the treatment for 6 to 9 weeks. Simultaneously, the doses of $0.15 \mathrm{and} 0.25 \mathrm{mg} / \mathrm{mL}$ PD and $200 \mathrm{mmol} / \mathrm{L}$ ethanol, determined by LDH efflux and mitochondrial respiration assays, were used to BRL-3A cells and not seen the drug toxicity. Simvastatin can improve the liver function and has protective effect against oxidative stress in damage liver via inducted Keap1-Nrf2 signal path (Habeos et al., 2008). In our study, the simvastatin was selected to find out the mechanism of PD on EFL.

It is show that the saponins, the aqueous extract and the PD from PG have hepatoprotective effects, and their mechanisms are via the activation of AMP-dependent protein kinase (Khanal et al.,2009) or TLR4-MyD88-NF- $\alpha B$ signal path (Wu et al., 2016). In our study, the PD is selected to learn the other possible mechanism of PD protection to liver injury.

The oxidative stress plays a role in the pathogenesis of ethanol-induced liver injury (Arteel, 2003), because of potentially toxic metabolic and biochemical processes of ethanol metabolism, including induction of CYP2E1 (Beier and Mcclain, 2010). CYP2E1 contributes to ethanol-induced oxidative stress and EFL in mice associating with increased generation of ROS (Lu et al., 2010; Gilmore et al., 2003). In our study, PD treatment decreased the levels of oxidative stress by decrease the levels of MDA, CYP2E1 and ROS in rats.

The body in response to the active oxygen damage has formed a complex oxidative stress response system, and the coordination response is regulated by ARE that is one of these protective genes in upstream regulation region (Itoh et al., 2003). Nrf2 is an activator of ARE, as a critical factor to regulate intracellular expression of many antioxidants, 
has play a role in keeping the balance between cellular oxidant and antioxidant. Anti-oxidation process of Nrf2 has closely related with various type of cellular factors that plays multiple protection role in the process (Stewart et al., 2003). Keapl-Nrf2-ARE, as an effective antioxidant regulation pathway, has played an important role for the defense, and reduced the oxidative stress of the human body (Rubiolo et al., 2008). When Keapl is under the stimulation of oxidation, its cysteine residues are oxidized, which result in the uncoupling of Nrf2 and Keapl, thereby to activate the phase detoxifying II enzyme gene in downstream elements of antioxidant response sequence to generate the gene transcription of antioxidant enzymes, such as HO-1, SOD and GSH-Px (Cai et al., 2005). Our data demonstrated that PD treatment inhibited the oxidative stress in ethanol-induced rats and BRL-3A cells, suggesting that the protective effect of the PD is via the activation of Keap1-Nrf2-ARE pathway.

\section{Conclusions}

The PD significantly activates signal path enzymes of Keap1, Nrf2 and small Maf either in vitro or in vivo, increases hepatic antioxidant enzyme expression, improves the oxidative stress, and then improves the extent of liver damage. The PD is proved to be an effective inhibitor of the EFL and the possible mechanism is that the PD treatment activates Keap1-Nrf2-ARE signal path and then enhances the body's antioxidant capacity.

Acknowledgements: This work was supported by the Dr. Fund Projects of University of Jinan under Grant XBS1321.

Conflict of Interest: The authors declare no conflict of interest.

\section{References}

1. Arteel, G.E. (2003). Oxidants and antioxidants in alcohol-induced liver disease. Gastroenterology. 124:778-790.

2. Beier, J.I., Mcclain, C.J. (2010). Mechanisms and cell signaling in alcoholic liver disease. Biological Chemistry, 391, 1249-1264.

3. Butura, A., Nilsson, K., Morgan, K., Morgan, T.R., French, S.W., Johansson, I., Schuppe-Koistinen, I., Ingelman-Sundberg, M. (2009). The impact of CYP2E1 on the development of alcoholic liver disease as studied in a transgenic mouse model. Journal of Hepatology. 50:572-583.

4. Cai, W.C., Wang, H., Li, R.Q., Gao, L. (2005). Determination of oxidation-reduction level in patients with rheumatoid arthritis. Journal of Southern Medical University. 25: 749-750.

5. Gilmore, W.J., Hartmann, G., Piquette-Miller, M., Marriott, J., Kirby, G.M. (2003). Effects of lipopolysaccharide-stimulated inflammation and pyrazole-mediated hepatocellular injury on mouse hepatic CYP2A5 expression. Toxicology. 184: 211-226.

6. Habeos, I.G., Ziros, P.G., Chartoumpekis, D., Psyrogiannis, A., Kyriazopoulou, V., Papavassiliou, A.G. (2008). Simvastatin activates keap1/Nrf2 signaling in rat liver. Journal of Molecular Medicine. 86: 1279-1285.

7. Itoh, K., Wakabayashi, N., Katoh, Y., Ishii, T., O'connor, T., Yamamoto, M. (2003). Keap1 regulates both cytoplasmic-nuclear shuttling and degradation of Nrf2 in response toelectrophiles. Genes to Cells. 8: 379-391.

8. Khanal, T., Choi, J. H., Hwang, Y. P., Chung, Y. C., Jeong, H. G. (2009). Protective effects of saponins from the root of platycodon grandiflorum against fatty liver in chronic ethanol feeding via the activation of amp-dependent protein kinase. Food \& Chemical Toxicology. 47: 2749-2754.

9. Lee, K. J., Jeong, H. G. (2002). Protective effect of platycodi radix on carbon tetrachloride-induced hepatotoxicity. Food \& Chemical Toxicology. 40:517-525.

10. Lee, K. J., You, H. J., Park, S. J., Kim, Y. S., Chung, Y. C., Jeong, T. C., Jeong, H G. (2001). Hepatoprotective effects of platycodon grandiflorum on acetaminophen-induced liver damage in mice. Cancer Letters, 174: 73-81.

11. Lu, Y., Wu, D., Wang, X., Ward, S.C., Cederbaum, A.I. (2010). Chronic ethanol-induced liver injury and oxidative stress are decreased in cytochrome P4502E1 knockout mice and restored in humanized cytochrome P4502E1 knock-in mice. Free Radical Biology \& Medicine. 49: 1406-1416.

12. National pharmacopoeia commission. (2015). Pharmacopoeia of the People's Republic of China, 2015 edition. China medical science and technology press: 750 .

13. Ngo, L.T., Okogun, J.I., Folk, W.R. (2013). 21st century natural product research and drug development and traditional medicines. Natural Product Reports. 30: 584-592.

14. Reisman, S.A., Aleksunes, L.M., Klaassen, C.D. (2009). Oleanolic acid activates Nrf2 and protects from acetaminophen hepatotoxicity via Nrf2-dependent and Nrf2-independent processes. Biochemical Pharmacology. 77, 1273-1282.

15. Rubiolo, J.A., Mithieux, G., Vega, F.V. (2008). Resveratrol protects primary rat hepatocytes against oxidative stress damage: Activation of the Nrf2 transcription factor and augmented activities of antioxidant enzymes. European Journal of Pharmacology. 591: 66-72.

16. Stewart, D., Killeen, E., Naquin, R., Alam, S., Alam, J. (2003). Degradation of transcription factor Nrf2 via the ubiquitin-proteasome pathway and stabilizationby cadmium. Journal of Biological Chemistry. 278: 396-402. 
17. Sussan, T.E., Rangasamy, T., Blake, D. J., Malhotra, D., Elhaddad, H., Bedja, D., Yates, M.S., Kombaeraju, P., Yamamoto, M., Liby, K.T., Sporn, M.B., Gabrielson, K.L. Champion, H.C., Tuder, R.M., Kensler, T.W., Biswal, S. (2009). Targeting Nrf2 with the triterpenoid CDDO-imidazolide attenuates cigarette smoke-induced emphysema and cardiac dysfunction in mice. Proceedings of the National Academy of Science. USA.106: 250-255.

18. Tong, K.I., Yasutake, K., Hideki, K., Ken, I., Toshiyuki, T., Masayuki, Y. (2006). Keap1 recruits Neh2 through binding to ETGE and DLG motifs: characterization of the two-site molecular recognition model. Molecular \& Cellular Biology. 26: 2887-2900.

19. Vishnudutt, P., Gao, B., Song, B. (2008). Molecular mechanisms of alcoholic fatty liver. Alcoholism Clinical \& Experimental Research. 33:191-205.

20. Wu, J.T., Yang, G.W., Qi, C.H., Zhou, L., Hu, J.G., Wang, M. S. (2016). Anti-inflammatory activity of platycodin D on alcohol-induced fatty liver rats via TLR4-Myd88-NF- $\mathrm{B}$ signal path. African Journal of Traditional Complementary \& Alternative Medicines. 13:176-183.

21. Wu, J.T., Yang, G.W., Zhu, W.X., Wen, W.J., Zhang, F.M., Yuan, J.D., An, L.G. (2012). Anti-atherosclerotic activity of platycodin D derived from roots of Platycodon grandiflorum in human endothelial cells. Biological \& Pharmaceutical Bulletin, 35: 1216-1221.

22. Yates, M.S., Kwak, M.K., Egner, P.A., Groopman, J.D., Bodreddigari, S., Sutter, T.R., Karen, J., Baumgartner, K.J., Roebuck, B.D., Liby, K.T., Yore, M.M., Honda, T., Gribble, G.W., Sporn, M.B., Kensler, T.W. (2006). Potent protection against aflatoxin-induced tumorigenesis through induction of Nrf2-regulated pathways by the triterpenoid 1-[2-cyano-3-, 12-dioxooleana-1, 9(11)-dien-28-oyl] imidazole. Cancer Research. 66: 2488-2494. 